\title{
Plasma Neutrophil Gelatinase-associated Lipocalin and Leukocyte Differential Count in Children with Febrile Urinary Tract Infection
}

\author{
Min Hwa Son, M.D. \\ Hyung Eun Yim, M.D., Ph.D. \\ Kee Hwan Yoo, M.D., Ph.D. \\ Department of Pediatrics, Korea \\ University College of Medicine, Seoul, \\ Korea \\ Corresponding author: \\ Hyung Eun Yim, MD, PhD \\ Address: Department of Pediatrics, \\ Korea University Ansan Hospital, Korea \\ University College of Medicine, 123 \\ Jeokgeum-ro, Danwon-gu, Ansan 15355, \\ Republic of Korea \\ Tel: +82-31-412-5096 \\ Fax: +82-31-405-8591 \\ E-mail: he-yim@hanmail.net \\ Received: 3 September 2021 \\ Revised: 18 October 2021 \\ Accepted: 16 November 2021
}

Purpose: We aimed to study the association of plasma neutrophil gelatinaseassociated lipocalin (pNGAL) and leukocyte differential count in children with febrile urinary tract infection (UTI).

Methods: Medical records of 154 children aged 1 month to 13 years with febrile UTI who were hospitalized were retrospectively reviewed. Associations between pNGAL levels and blood leukocyte differential count at admission and after 48 hours of treatment were investigated in children with or without acute pyelonephritis (APN).

Results: The APN group ( $n=82$ ) showed higher pNGAL levels, neutrophil count, monocyte count, and neutrophil-to-lymphocyte ratio (NLR), compared to the non-APN group ( $\mathrm{n}=72$ ) (all $P<0.05)$. After adjustment for age and sex, pNGAL showed positive correlations with neutrophil count and NLR in both groups (all $P<0.05)$. Additionally, it was correlated with the monocyte-to-lymphocyte ratio (MLR) only in the APN group $(P<0.05)$. Before and after treatment, pNGAL was positively correlated with neutrophil count, NLR, and MLR in patients with APN while it was related with neutrophil count and NLR in those without APN (all $P<$ 0.05). Areas under the receiver operating curve of pNGAL, neutrophil count, NLR, and MLR for predicting APN were $0.804,0.760,0.730$, and 0.636 , respectively (all $P<0.05)$. Only pNGAL was independently associated with the presence of APN in a multivariable logistic regression analysis $(P<0.05)$.

Conclusion: In children with febrile UTIs, pNGAL might be associated with leukocyte differential count and the presence of APN.

Key words: Biomarkers, Lipocalin-2, Pyelonephritis

\section{Introduction}

Urinary tract infection (UTI) is one of the most common diseases in the first year of life after birth in both male and female infants ${ }^{1}$. Delayed diagnosis and treatment of UTI can increase the risk of sepsis in newborns ${ }^{2}$. The spread of infection to kidneys can cause renal damage and chronic kidney disease (CKD) in the long term ${ }^{3)}$. Therefore, prompt diagnosis and immediate treatment for UTI are essential. Numerous studies have been conducted recently to find ways to promptly diagnose UTI. In addition, several imaging tests such as kidney ultrasonography, renal scan, and voiding cystourethrography (VCUG) have been introduced to identify congenital anomalies of the
This is an open-access article distributed under the terms of the Creative Commons Attribution Non-Commercial License (http:// creativecommons.org/licenses/by-nc/4.0/) which permits unrestricted non-commercial use, distribution, and reproduction in any medium, provided the original work is properly cited.

Copyright (C) 2021 The Korean Society of Pediatric Nephrology 
kidney and urinary tract (CAKUT), acute pyelonephritis (APN), and consequent kidney scarring ${ }^{4,5}$. However, renal scan and VCUG tests are invasive with a risk of irradiation.

It is generally believed that neutrophil leukocytosis is associated with bacterial infections while lymphocytosis is associated with viral infections ${ }^{6}$, although such associations are not absolute. It has been reported that neutrophils in first responder cells are essential for survival because of their ability to contain and eliminate bacterial and fungal pathogens ${ }^{7}$. Monocytes are also involved in the elimination of pathogens and dead cells in tissues. They are also involved in the initiation of adaptive immunity ${ }^{8}$. Environ. mental signals at the inflamed site can mediate rapid monocyte recruitment, directing a differentiation program in which monocytes can trigger macrophages or dendritic cells. It has shown that the neutrophil-to-lymphocyte ratio (NLR) and the monocyte-to-lymphocyte ratio (MLR) are useful for diagnosing bacterial infections in febrile patients ${ }^{6,9-11)}$. Han et al. ${ }^{10)}$ have reported that NLR is a predictive factor for detecting APN in young children with febrile UTI. Wang et al. ${ }^{11)}$ have discovered that MLR is an independent risk factor for a serious Klebsiella pneumonia infection. Compared to NLR, MLR is a better indicator for the severity of Klebsiella pneumonia infection ${ }^{11}$.

Neutrophil gelatinase-associated lipocalin (NGAL), also called lipocalin 2, is an iron carrier protein that is abundantly expressed in neutrophils and macrophages. It plays a role in mediating innate immune responses of mammals ${ }^{12)}$. Earlier studies have demonstrated that NGAL level could be useful for diagnosing APN in children with febrile UTI and that it might be an alternative to imaging tests for pediatric $\mathrm{UTI}^{13,14)}$. Additionally, we have previously found that urine NGAL (uNGAL) levels are positively correlated with neutrophil count, monocyte count, NLR, and MLR in children with $\mathrm{UTI}^{15}$. However, the association of plasma NGAL (pNGAL) levels with leukocyte differential count such as NLR and MLR in children with febrile UTIs remains unknown. Thus, the objective of this study was to determine whether plasma NGAL (pNGAL) levels were associated with leukocyte differential count, especially NLR and MLR, in children with febrile UTIs. Values of NLR, MLR, and NGAL for predicting acute pyelonephritis (APN) in children with febrile UTIs were also validated.

\section{Material and methods}

\section{Patient characteristics and inclusion criteria}

This was a retrospective observational study of patients who were hospitalized in the Department of Pediatrics and Adolescents at our institution from August 21, 2018 to October 20, 2019 due to febrile UTI. Patients aged 1 month to 13 years were included in this study. UTI was defined based on revised guidelines of the American Academy of Pediatrics ${ }^{16}$. Patients were defined to have APN if the uptake of 99mTc-dimercaptosuccinic acid (DMSA) 1-5mCi was decreased in a focal, multifocal, or diffuse area due to defects in the renal cortex ${ }^{17)}$.

Inclusion criteria of this study were as follows: i) hospitalized with fever $\left(\geq 38^{\circ} \mathrm{C}\right)$; ii) positive results on urine analysis for pyuria or nitrite; and iii) $\geq 100,000 / C F U$ for a single bacterium on urine culture through urethral catheterization in non-toilet-trained infants or midstream urine in toilet-trained children. Patients were excluded if they had any of the following conditions: i) below 1 month of age; ii) recurrent UTI; iii) acute kidney injury (AKI); iv) CKD; v) systemic disease, vi) CAKUT except vesicoureteral reflux (VUR).

Excluded systemic diseases included coincidental viral or mycoplasma infection, Budd-Chiari syndrome, chronic respiratory failure, and others (Fig. 1). Since NGAL is upregulated in systemic inflammatory response syndrome and systemic diseases without bacterial infection as well as in renal tubular injury ${ }^{18}$, the aforementioned groups of patients were not included in this study. Newborns were also excluded from this study due to the immature and plastic nature of neonatal immune response.

We divided patients with UTI into APN and non-APN groups and compared absolute neutrophil count (ANC), lymphocyte count, monocyte count, NLR, and MLR between the two groups. The association between pNGAL and leukocyte differential count was investigated in both APN and non-APN groups. Patients were usually hospitalized for about 7 days. They were discharged when there were no clinical symptoms with the absence of bacteria confirmed by a urine culture test.

\section{Laboratory and radiological assessment}

Hospitalized children underwent urine tests and basic 
blood tests including pNGAL level, white blood cell (WBC) count, ANC, lymphocyte count, monocyte count, and Creactive protein (CRP) level.

Levels of pNGAL were measured after centrifuging plasma samples at $2,100 \mathrm{~g}$ for $10 \mathrm{~min}$ at $10^{\circ} \mathrm{C}$ in ethylenediaminetetraacetic acid using a Cobas 8000 c702 (Roche Diagnostics, Rotkreuz, Switzerland). NGAL Test ${ }^{\mathrm{TM}}$ (Bioporto Diagnostics, Denmark) reagent was used for the measurement. It had a detection range of $25-3,000 \mathrm{ng} / \mathrm{mL}$. For urinalysis, we used a Sysmex UF-1000i ${ }^{\mathrm{TM}}$ Urine Cell Analyzer System and an Arkray, a fully automated urine analyzer. Urine samples were immediately centrifuged at 494 $\mathrm{g}$ for $5 \mathrm{~min}$ at $10^{\circ} \mathrm{C}$. Patients with febrile UTIs underwent kidney ultrasonography and DMSA scan. When necessary, VCUG was performed on the day before discharge.

\section{Statistical analysis}

IBM SPSS ver. 20.0 (IBM Co., Armonk, NY, USA) was used for all statistical analyses. For comparison between APN and non-APN groups, Mann-Whitney U test was used for continuous variables and Chi-square test was used for categorical variables. Continuous variables are presented as mean \pm standard deviation or standard error of the mean. Categorical variables are presented as number (\%). Spearman partial correlation coefficient was used to find relationships of pNGAL levels with WBC, absolute neutrophil count (ANC), monocyte, NLR, and MLR after adjusting for age and sex. Univariable logistic regression analyses were performed to analyze variables associated with the presence of APN. Parameters associated with APN in univariable logistic regression analyses $(P<0.05)$ were included in a multivariable logistic regression analysis. Receiver operating curve (ROC) analyses were performed to determine how accurately pNGAL level and leukocyte differential count could discriminate between APN and non-APN. The area under the curve (AUC) with 95\% confidence intervals (CIs) was calculated. In all statistical analyses, $P<0.05$ was considered statistically significant.

\section{Ethics statement}

This study was approved by the Institutional Review Board (IRB) and the Research Ethics Committee of Korea University Ansan Hospital [IRB number: 2019AS0155]. The IRB exempted the requirement of informed consent because of the retrospective nature of this study. Personal identifiers were completely removed and data were analyzed anonymously. This study was performed in accordance with the Declaration of Helsinki.

\section{Results}

\section{Patient demographics}

A total of 233 patients were hospitalized for UTI. Of them, 79 were excluded from this study. Of the remaining 154 patients, 82 had APN while 72 did not (Fig. 1). There was no significant differences in age or sex between APN and non-APN groups. However, the period of hospitalization was longer in the APN group (APN vs. non-APN: 7.27 \pm 1.58 days vs. $6.42 \pm 1.46$ days, $P<0.05)$. In addition, the APN group had more cases with fever that persisted for $\geq 72$ hours after onset of symptoms (APN vs. non-APN, 46.3\% vs. $20.8 \%, P<0.05)$. Levels of pNGAL, erythrocyte sedimentation rate, CRP, WBC, ANC, monocyte count, and NLR were significantly higher in the APN group than in the non-APN group (all $P<0.05$ ). However, there was no significant difference in creatinine, lymphocyte, or MLR between the two groups. In all patients, serum creatinine levels were within the normal range for their age (Table 1).

\section{Association between pNGAL level and leukocyte differential count}

Age and sex-adjusted Spearman correlation analysis was performed to find out the relationship between pNGAL level and leukocyte differential count in both APN and

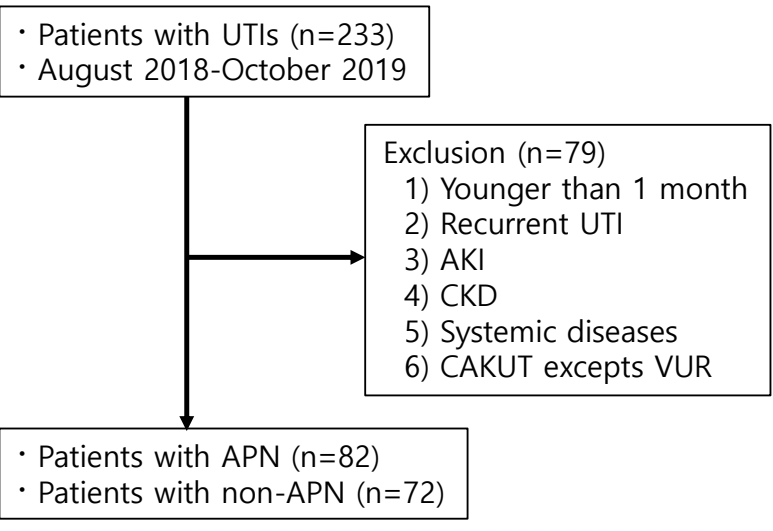

Fig. 1. Study flow diagram. 
non-APN groups. In the APN group, WBC, ANC, NLR, and MLR were associated with pNGAL level (all $P<0.05)$. In the non-APN group, WBC, ANC, and NLR were associated with pNGAL level (all $P<0.05$ ) (Table 2 ). However, levels of pNGAL were not fully available for all patients because there were cases when the test was not done on the first day of admission.

Table 1. Patient Characteristics and Clinical Findings

\begin{tabular}{lccr}
\hline & $\begin{array}{c}\text { APN } \\
\mathrm{N}=82\end{array}$ & $\begin{array}{c}\text { non-APN } \\
\mathrm{N}=72\end{array}$ & P-value \\
\hline Male, $\mathrm{n}(\%)$ & $54(65.9 \%)$ & $45(62.5 \%)$ & $0.791^{*}$ \\
Age (month) & $10.8 \pm 23.5$ & $8.54 \pm 15.1$ & $0.482^{\dagger}$ \\
Admission day & $7.3 \pm 1.6$ & $6.4 \pm 1.5$ & $0.001^{\dagger}$ \\
Fever duration (> 72 hrs), & $38(46.3 \%)$ & $15(20.8 \%)$ & $0.002^{*}$ \\
n (\%) & & & \\
pNGAL (ng/mL) & $176.0 \pm 141.0$ & $82.1 \pm 59.0$ & $<0.001^{\dagger}$ \\
Cr (mg/dL) & $0.27 \pm 0.09$ & $0.24 \pm 0.06$ & $0.075^{\dagger}$ \\
ESR (mm/hr) & $35.4 \pm 20.6$ & $21.7 \pm 18.3$ & $<0.001^{\dagger}$ \\
CRP (mg/L) & $5.72 \pm 4.65$ & $2.56 \pm 2.66$ & $<0.001^{\dagger}$ \\
WBC (/mm $\left.{ }^{3}\right)$ & $17,975 \pm 5,878$ & $13,509 \pm 5,243$ & $<0.001^{\dagger}$ \\
ANC (/mm $\left.{ }^{\dagger}\right)$ & $10,163 \pm 4766$ & $6,366 \pm 3751$ & $<0.001^{\dagger}$ \\
Lymphocyte count $\left(/ \mathrm{mm}^{3}\right)$ & $5,722 \pm 2,304$ & $5,489 \pm 2,333$ & $0.534^{\dagger}$ \\
Monocyte count $\left(/ \mathrm{mm}^{3}\right)$ & $1,908 \pm 1,037$ & $1,430 \pm 738$ & $0.001^{\dagger}$ \\
NLR & $2.43 \pm 3.93$ & $1.35 \pm 1.05$ & $0.019^{\dagger}$ \\
MLR & $0.38 \pm 0.26$ & $0.31 \pm 0.25$ & $0.1^{\dagger}$ \\
\hline
\end{tabular}

${ }^{*}$ Chi-square test; ${ }^{\dagger}$ Mann-Whitney $U$ test.

Values are presented as mean \pm standard deviation or number (\%).

Abbreviations: APN, acute pyelonephritis; pNGAL, plasma neutrophil gelatinase-associated lipocalin; BUN, blood urea nitrogen; $\mathrm{Cr}$, creatinine; ESR, Erythrocyte sedimentation rate; CRP, C-reactive protein; WBC, white blood cell; ANC, absolute neutrophil count; NLR, neutrophil-to-lymphocyte ratio; $M L R$, monocyte-to-lymphocyte ratio.

Table 2. Spearman Correlation Analysis between pNGAL Level and Leukocyte Differential Count after Adjusting for Age and Sex

\begin{tabular}{lcccccc}
\hline & \multicolumn{3}{c}{ APN } & & \multicolumn{2}{c}{ non-APN } \\
\cline { 2 - 3 } \cline { 5 - 6 } & Spearman's $\rho$ & $P$-value & & Spearman's $\rho$ & $P$-value \\
\hline ANC $(/ \mathrm{mm})$ & 0.396 & 0.001 & & 0.421 & 0.002 \\
Monocyte $\left(/ \mathrm{mm}^{3}\right)$ & -0.008 & 0.951 & & -0.031 & 0.827 \\
WBC $\left(/ \mathrm{mm}^{3}\right)$ & 0.293 & 0.019 & & 0.37 & 0.008 \\
NLR & 0.444 & $<0.001$ & & 0.402 & 0.004 \\
MLR & 0.295 & 0.018 & & 0.157 & 0.271 \\
\hline
\end{tabular}

Abbreviations: Spearman's $\rho$, Spearman partial correlation coefficient; pNGAL, plasma neutrophil gelatinase-associated lipocalin; APN, acute pyelonephritis; ANC, absolute neutrophil count; WBC, white blood cell; NLR, neutrophil-to-lymphocyte ratio; MLR, monocyte-to-lymphocyte ratio.

\section{Univariable and multivariable logistic regression} analyses for APN in patients with UTI

In univariable logistic analyses, pNGAL, WBC, CRP, ANC, monocyte count, and NLR were associated with the presence of APN in children with febrile UTIs (all $P<0.05$ ). Among these variables, only pNGAL level was an independent predictor for the presence of APN in a multivariable logistic regression analysis $(P<0.05)$ (Table 3$)$.

\section{ROC analyses}

ROC analysis was performed to find the most appropriate parameter for detecting APN in febrile UTIs. Results revealed that pNGAL level had the highest AUC $(0.804,95 \%$ CI: $0.721-0.871)$, followed by WBC (0.721, 95\% CI: $0.644-$ 0.791), ANC (0.760, 95\% CI: 0.669-0.812), NLR (0.730, 95\% CI: 0.614-0.765), MLR (0.636, 95\% CI: 0.529-0.688), and CRP (0.741, 95\% CI: 0.652-0.817) (all $P<0.05$ ) (Fig. 2). The best cut-off value of pNGAL for detecting APN was 94.3 $\mathrm{ng} / \mathrm{mL}$, with a sensitivity of $72.7 \%$ and a specificity of 79.3 $\%$. The best cut-off value of $\mathrm{WBC}$ was $15,310 / \mathrm{mm}^{3}$, with a sensitivity of $69.5 \%$ and a specificity of $68.1 \%$. The best cutoff value of ANC was $7,031 / \mathrm{mm}^{3}$, with a sensitivity of 75.6 $\%$ and a specificity of $66.7 \%$. The best cut-off value of NLR was 1.53 , with a sensitivity of $58.5 \%$ and a specificity of 75 $\%$. The best cut-off value of MLR was 0.35 , with a sensitivity of $46.3 \%$ and a specificity of $77.8 \%$. The best cut-off value

Table 3. Univariable and Multivariable Logistic Regression Analyses for APN in Patients with UTI

\begin{tabular}{|c|c|c|c|c|}
\hline \multirow{2}{*}{ Variables } & \multicolumn{2}{|c|}{$\begin{array}{c}\text { Univariable logistic } \\
\text { regression }\end{array}$} & \multicolumn{2}{|c|}{$\begin{array}{c}\text { Multivariable logistic } \\
\text { regression }\end{array}$} \\
\hline & $\begin{array}{l}\text { Odds ratio } \\
(95 \% \mathrm{Cl})\end{array}$ & $\begin{array}{c}P_{-} \\
\text {value }\end{array}$ & $\begin{array}{l}\text { Odds ratio } \\
(95 \% \mathrm{Cl})\end{array}$ & $\begin{array}{c}P_{-} \\
\text {value }\end{array}$ \\
\hline Age (month) & $1.01(0.99-1.02)$ & 0.497 & $0.99(0.96-1.03)$ & 0.749 \\
\hline Sex (male) & $0.87(0.44-1.68)$ & 0.669 & $0.59(0.23-1.47)$ & 0.264 \\
\hline pNGAL (ng/mL) & $1.02(1.01-1.02)$ & $<0.001$ & $1.01(1.00-1.02)$ & 0.033 \\
\hline WBC $\left(/ \mathrm{mm}^{3}\right)$ & $1.00(1.00-1.00)$ & $<0.001$ & $1.00(1.00-1.00)$ & 0.661 \\
\hline CRP (mg/L) & $1.31(1.16-1.48)$ & $<0.001$ & $1.14(0.96-1.37)$ & 0.146 \\
\hline ANC $\left(/ \mathrm{mm}^{3}\right)$ & $1.00(1.00-1.00)$ & $<0.001$ & $1.00(1.00-1.00)$ & 0.854 \\
\hline Lymphocyte $\left(/ \mathrm{mm}^{3}\right)$ & $1.00(1.00-1.00)$ & 0.532 & & \\
\hline Monocyte $\left(/ \mathrm{mm}^{3}\right)$ & $1.00(1.00-1.00)$ & 0.002 & $1.00(1.00-1.00)$ & 0.463 \\
\hline NLR & $1.72(1.23-2.42)$ & 0.002 & $1.21(0.80-3.73)$ & 0.728 \\
\hline MLR & $3.21(0.76-13.5)$ & 0.112 & & \\
\hline
\end{tabular}

Abbreviations: APN, acute pyelonephritis; UTI, urinary tract infection; pNGAL, plasma neutrophil gelatinase-associated lipocalin; WBC, white blood cell; CRP, C-reactive protein; ANC, absolute neutrophil count; NLR, neutrophil-to-lymphocyte ratio; $M L R$, monocyte-to-lymphocyte ratio. 
of CRP was 2.1, with a sensitivity of $76.8 \%$ and a specificity of $58.3 \%$ (Table 4 ).

\section{Discussion}

The aim of this study was to assess the relationship between pNGAL level and leukocyte differential count in children with febrile UTIs. pNGAL level was found to be correlated with ANC and NLR in both APN and non-APN groups. In particular, it had an association with MLR in

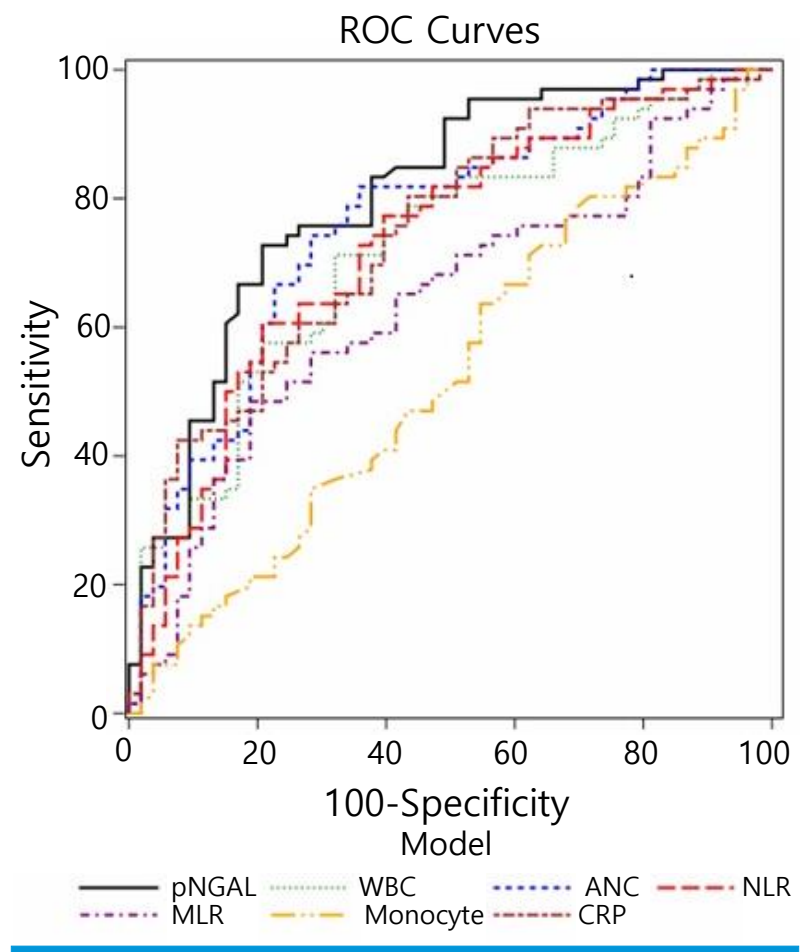

Fig. 2. Receiver operating curves for finding a reliable predictive biomarker for APN. the APN group, but not in the non-APN group. Results of logistic regression analysis confirmed that only pNGAL level could predict the presence of APN independently in children with febrile UTIs. For predicting APN, pNGAL level was found to have the highest AUC value among examined variables. Therefore, pNGAL might be associated with neutrophil count and NLR in febrile UTI. In addition, it was associated with MLR, especially in those with APN. We also confirmed that pNGAL could be a useful tool for diagnosing APN in children with febrile UTIs.

The migration of leukocytes is a strictly regulated process that controls the distribution of immune cells throughout the body ${ }^{19)}$. Upon infection, neutrophils and monocytes move to the peripheral site. They are essential for the defense against a number of microbial pathogens ${ }^{20)}$. In a normal condition, these cells are rarely present in peripheral tissues. However, if an infection occurs, numbers of neutrophils and monocytes are increased rapidly. They can proliferate in the bone marrow and migrate to the site of the infection or inflammation ${ }^{20)}$. Recent studies have shown that neutrophils are involved in both protective and detrimental responses to various types of inflammation and infectious diseases $^{7)}$. Condron et al. ${ }^{21)}$ have suggested that a defective host neutrophil response can lead to a susceptibility to recurrent UTI. Neutrophils from recurrent UTI patients show a significant decrease in bactericidal function compared to those in healthy controls, with the former demonstrating a decrease in activation capacity ${ }^{21)}$. This finding implies that a reduced function of neutrophils can result in an inadequate bacterial eradication and an increased susceptibility to recurrent infections. Likewise, monocytes play an important role in the initiation of adaptive immunity, the removal of pathogens and dead cells, and tissue healing ${ }^{8)}$.

Table 4. Predictive Values of Biomarkers for APN Based on ROC Curve Analysis

\begin{tabular}{|c|c|c|c|c|c|c|}
\hline & AUC & $95 \% \mathrm{Cl}$ & $P$-value & Cut-off value & Sensitivity (\%) & Specificity (\%) \\
\hline pNGAL (ng/mL) & 0.804 & $0.721-0.871$ & $<0.0001$ & 94.3 & 72.7 & 79.3 \\
\hline WBC $\left(/ \mathrm{mm}^{3}\right)$ & 0.721 & $0.644-0.791$ & $<0.0001$ & 15,310 & 69.5 & 68.1 \\
\hline ANC $\left(/ \mathrm{mm}^{3}\right)$ & 0.760 & $0.669-0.812$ & $<0.0001$ & 7,031 & 75.6 & 66.7 \\
\hline Monocyte $\left(/ \mathrm{mm}^{3}\right)$ & 0.527 & $0.437-0.600$ & 0.680 & 13 & 75.6 & 30.6 \\
\hline NLR & 0.730 & $0.614-0.765$ & $<0.0001$ & 1.53 & 58.5 & 75.0 \\
\hline MLR & 0.636 & $0.529-0.688$ & 0.016 & 0.35 & 46.3 & 77.8 \\
\hline CRP (mg/L) & 0.741 & $0.652-0.817$ & $<0.0001$ & 2.1 & 76.8 & 58.3 \\
\hline
\end{tabular}

Abbreviations: APN, acute pyelonephritis; ROC, Receiver operating curve; AUC, area under the curve; $\mathrm{Cl}$, confidence interval; pNGAL, plasma neutrophil gelatinase-associated lipocalin; WBC, white blood cell; ANC, absolute neutrophil count; NLR, neutrophil-to-lymphocyte ratio; MLR, monocyte-to-lymphocyte ratio; CRP, C-reactive protein. 
Monocytes recruited into the tissue can mediate defense mechanism against pathogens and contribute to inflammatory reactions. Thus, regulation of circulating monocytes are crucial in controlling disease progression as well as host defense from invading pathogens ${ }^{19)}$. Naess et al. ${ }^{6}$ ) have reported that both NLR and MLR are effective for diagnosing bacterial infections in hospitalized patients with fever and that NLR is more useful than commonly used diagnostic blood tests for diagnosing septicemia. Jager et al. $^{22)}$ have reported that NLR and lymphocytopenia are better markers for predicting bacteremia than conventional infection markers such as CRP, WBC, and neutrophil count. In our previous study, we have found that children with UTIs show higher monocyte counts than those with viral infections ${ }^{15)}$. Monocyte count has been found to be more specific than any other indices of leukocyte differential count for detecting UTIs in children with fever ${ }^{15)}$. In the present study, ANC, monocyte count, NLR, and pNGAL level were significantly higher in the APN group than in the non-APN group. These results suggest that neutrophils and monocytes might play a role in the presence and severity of UTI in children with fever.

NGAL is an iron-carrier protein produced by neutrophil granules that play a vital role in the innate immune system against bacterial infections ${ }^{23,24)}$. When there is an infection, bacteria require iron for growth and metabolism in the host. Bacteria acquire much of their iron from the host by synthesizing siderophores that can remove iron and transport it into the pathogen ${ }^{25,26)}$. This leads to activation of neutrophils of the host to release NGAL, which prevents bacteria from absorbing iron ${ }^{24,27)}$. NGAL transcription is induced by bacterial lipopolysaccharides via a Toll-like receptor. Bacterial proliferation is inhibited by the depletion of iron-laden siderophores such as enterochelin ${ }^{28)}$. Elevated pNGAL levels have been reported in patients with community-acquired pneumonia (CAP), sepsis, bacterial meningitis, peritonitis, and $\mathrm{UTI}^{29)}$. pNGAL level is positively correlated with CRP and procalcitonin levels in patients with $\mathrm{CAP}^{30)}$. It shows the highest AUC value for predicting mortality of $\mathrm{CAP}^{30)}$. In another paper, lipocalin 2 concentration was significantly higher in patients with acute bacterial meningitis than in those with viral meningitis ${ }^{31)}$. We have repeatedly shown that NGAL could be a useful marker for detecting and managing UTI and APN in children ${ }^{4,14}$,
${ }^{32-34)}$. Increased pNGAL levels are associated with the presence of APN and subsequent kidney scarring in children with febrile UTIs ${ }^{14,32,33)}$. Recently, we have found that uNGAL level is positively correlated with ANC, monocyte count, NLR, and MLR in children with UTIs ${ }^{15)}$. Both monocyte count and uNGAL level are useful for distinguishing between UTI and non-UTI groups ${ }^{15)}$. In the present study, pNGAL level was correlated with ANC and NLR in all patients with UTI after adjusting for age and sex. pNGAL level also showed an association with MLR in the APN group. MLR was associated with pNGAL level in the APN group. These findings imply that ANC, NLR, and NGAL might play a role in managing children with febrile UTI and that MLR might be useful for assessing children with APN.

In the present study, only pNGAL level was an independent predictor for APN in children with febrile UTIs when pNGAL, WBC, CRP, ANC, monocyte count, and NLR were included in a multivariable logistic analysis. ROC analyses also showed that pNGAL level had the highest AUC value for predicting APN, followed by ANC, NLR, WBC, and MLR. These findings were consistent with our previous findings ${ }^{14,15)}$. Krzemien et al. ${ }^{13)}$ have reported that pNGAL level is a good marker for early diagnosis of UTIs in febrile infants and that it has higher sensitivity and specificity than uNGAL. Other studies have also suggested that pNGAL might be a sensitive predictor to identify APN and monitor treatment response in pediatric $\mathrm{UTIs}^{32,33)}$.

APN can progress to life-threatening sepsis and multiorgan failure. When accompanied by sepsis, the risk of mortality and long-term hospitalization dramatically increases and several complications can occur ${ }^{35-37)}$. As APN is one of serious bacterial infections that can cause kidney scarring in children. Thus, early diagnosis and prompt treatment are crucial. When NGAL measurement is unavailable, monitoring the level of neutrophil count or NLR in children with suspected UTIs may be useful. MLR can be additionally helpful for predicting APN in febrile UTIs. Taken together, there might be a correlation between NGAL and leukocyte differential count in patients with febrile UTIs.

This study has some limitations. First, this study was performed in a single institution with a relatively small sample size. In addition, this was a retrospective study. Some child- 
ren took oral antibiotics before their hospitalization. In some children, WBC counts in the initial urine analysis were normal. In those cases, children were monitored without intravenous antibiotic treatment until urine culture reports showed that bacteria were growing, usually the second day of hospitalization. Lastly, pNGAL data were not collected if UTI was not suspected in the initial presentation. Patients with initial pNGAL values missing were excluded from some analysis.

In conclusion, pNGAL level is associated with blood leukocyte differential count in children with febrile UTIs. While ANC and NLR could be helpful for diagnosing febrile UTIs, MLR would be better for diagnosing APN in children with UTI. Additional studies involving multiple centers with a large sample size are needed to clarify the association of leukocyte differential count with NGAL level in children with suspected UTIs.

\section{Conflict of interest}

No potential conflict of interest relevant to this article was reported.

\section{ORCID}

Min Hwa Son https://orcid.org/0000-0002-4185-1712

Hyung Eun Yim https://orcid.org/0000-0001-9805-9278

Kee Hwan Yoo https://orcid.org/0000-0001-6490-4293

\section{References}

1) Montini G, Tullus $K$, Hewitt I. Febrile urinary tract infections in children. N Engl J Med 2011;365:239-50.

2) Morello W, La Scola C, Alberici I, Montini G. Acute pyelonephritis in children. Pediatr Nephrol 2016;31:1253-65.

3) Yilmaz A, Sevketoglu E, Gedikbasi A, Karyagar S, Kiyak A, Mulazimoglu $\mathrm{M}$, et al. Early prediction of urinary tract infection with urinary neutrophil gelatinase associated lipocalin. Pediatr Nephrol 2009;24:2387-92.

4) Yim HE, Yim H, Bae ES, Woo SU, Yoo KH. Predictive value of urinary and serum biomarkers in young children with febrile urinary tract infections. Pediatr Nephrol 2014;29:2181-9.
5) Kass EJ, Kernen KM, Carey JM. Paediatric urinary tract infection and the necessity of complete urological imaging. BJU Int 2000; 86:94-6.

6) Naess A, Nilssen SS, Mo R, Eide GE, Sjursen H. Role of neutrophil to lymphocyte and monocyte to lymphocyte ratios in the diagnosis of bacterial infection in patients with fever. Infection 2017; 45:299-307.

7) Witter AR, Okunnu BM, Berg RE. The Essential role of neutrophils during infection with the intracellular bacterial pathogen Listeria monocytogenes. J Immunol 2016;197:1557-65.

8) Ingersoll MA, Platt AM, Potteaux S, Randolph GJ. Monocyte trafficking in acute and chronic inflammation. Trends Immunol 2011; 32:470-7.

9) Forget $P$, Khalifa C, Defour JP, Latinne D, Van Pel MC, De Kock M. What is the normal value of the neutrophil-to-lymphocyte ratio? BMC Res Notes 2017;10:12.

10) Han SY, Lee IR, Park SJ, Kim JH, Shin JI. Usefulness of neutrophillymphocyte ratio in young children with febrile urinary tract infection. Korean J Pediatr 2016;59:139-44.

11) Wang JL, Lu XY, Xu XH, Zhang KJ, Gong H, Lv D, et al. Predictive role of monocyte-to-lymphocyte ratio in patients with Klebsiella pneumonia infection: a single-center experience. Medicine (Baltimore) 2019;98:e17215.

12) Ichino M, Kuroyanagi Y, Kusaka M, Mori T, Ishikawa K, Shiroki R, et al. Increased urinary neutrophil gelatinase associated lipocalin levels in a rat model of upper urinary tract infection. J Urol 2009; 181:2326-31.

13) Krzemien G, Panczyk-Tomaszewska M, Adamczuk D, Kotula I, Demkow U, Szmigielska A. Neutrophil gelatinase-associated lipocalin: a biomarker for early diagnosis of urinary tract infections in infants. Adv Exp Med Biol 2018;1047:71-80.

14) Kim BK, Yim HE, Yoo KH. Plasma neutrophil gelatinase-associated lipocalin: a marker of acute pyelonephritis in children. Pediatr Nephrol 2017;32:477-84.

15) Jang JW, Yim HE, Yoo KH. Association of neutrophil gelatinase associated lipocalin and leukocyte differential count in children with febrile urinary tract infections. Child Kidney Dis 2020;24:8390 .

16) Subcommittee on Urinary Tract Infection SCoQI, Management, Roberts KB. Urinary tract infection: clinical practice guideline for the diagnosis and management of the initial UTI in febrile infants and children 2 to 24 months. Pediatrics 2011;128:595-610.

17) Jaksic E, Bogdanovic R, Artiko V, Saranovic DS, Petrasinovic Z, Petrovic $M$, et al. Diagnostic role of initial renal cortical scintigraphy in children with the first episode of acute pyelonephritis. Ann Nucl Med 2011;25:37-43.

18) Schmidt-Ott KM, Mori K, Li JY, Kalandadze A, Cohen DJ, Devarajan $P$, et al. Dual action of neutrophil gelatinase-associated lipocalin. J Am Soc Nephrol 2007;18:407-13.

19) Serbina NV, Pamer EG. Monocyte emigration from bone marrow during bacterial infection requires signals mediated by chemokine receptor CCR2. Nat Immunol 2006;7:311-7. 


\section{www.chikd.org}

20) Serbina NV, Hohl TM, Cherny M, Pamer EG. Selective expansion of the monocytic lineage directed by bacterial infection. J Immunol 2009;183:1900-10.

21) Condron C, Toomey D, Casey RG, Shaffii M, Creagh T, BouchierHayes D. Neutrophil bactericidal function is defective in patients with recurrent urinary tract infections. Urol Res 2003;31:329-34.

22) de Jager CP, van Wijk PT, Mathoera RB, de Jongh-Leuvenink J, van der Poll T, Wever PC. Lymphocytopenia and neutrophil-lymphocyte count ratio predict bacteremia better than conventional infection markers in an emergency care unit. Crit Care 2010;14:1-8.

23) Xu SY, Carlson M, Engstrom A, Garcia R, Peterson CG, Venge P. Purification and characterization of a human neutrophil lipocalin (HNL) from the secondary granules of human neutrophils. Scand J Clin Lab Invest 1994;54:365-76.

24) Berger T, Togawa A, Duncan GS, Elia AJ, You-Ten A, Wakeham A, et al. Lipocalin 2-deficient mice exhibit increased sensitivity to Escherichia coli infection but not to ischemia-reperfusion injury. Proc Natl Acad Sci USA 2006;103:1834-9.

25) Ratledge C, Dover LG. Iron metabolism in pathogenic bacteria. Annu Rev Microbiol 2000;54:881-941.

26) Faraldo-Gomez JD, Sansom MS. Acquisition of siderophores in gram-negative bacteria. Nat Rev Mol Cell Biol 2003;4:105-16.

27) Nguyen MT, Devarajan P. Biomarkers for the early detection of acute kidney injury. Pediatr Nephrol 2008;23:2151-7.

28) Flo TH, Smith KD, Sato S, Rodriguez DJ, Holmes MA, Strong RK, et al. Lipocalin 2 mediates an innate immune response to bacterial infection by sequestrating iron. Nature 2004;432:917-21.

29) Nasioudis D, Witkin SS. Neutrophil gelatinase-associated lipocalin
Son MH, et al. • Plasma NGAL and Leukocyte Differential Count

and innate immune responses to bacterial infections. Med Microbiol Immunol 2015;204:471-9.

30) Kim JW, Hong DY, Lee KR, Kim SY, Baek KJ, Park SO. Usefulness of plasma neutrophil gelatinase-associated lipocalin concentration for predicting the severity and mortality of patients with community-acquired pneumonia. Clin Chim Acta 2016;462:140-5.

31) Guiddir T, Deghmane AE, Giorgini D, Taha MK. Lipocalin 2 in cerebrospinal fluid as a marker of acute bacterial meningitis. BMC Infect Dis 2014;14:1-8.

32) Sim JH, Yim HE, Choi BM, Lee JH, Yoo KH. Plasma neutrophil gelatinase-associated lipocalin predicts acute pyelonephritis in children with urinary tract infections. Pediatr Res 2015;78:48-55.

33) Lee JH, Yim HE, Yoo KH. Associations of plasma neutrophil gelatinase-associated lipocalin, anemia, and renal scarring in children with febrile urinary tract infections. J Korean Med Sci 2020;35:e65.

34) Moon JH, Yoo KH, Yim HE. Urinary neutrophil gelatinase-associated lipocalin: a marker of urinary tract infection among febrile children. Clin Exp Pediatr 2020.

35) Kim B, Myung R, Kim J, Lee MJ, Pai H. Descriptive epidemiology of acute pyelonephritis in Korea, 2010-2014: Population-based Study. J Korean Med Sci 2018;33:e310.

36) Elkharrat D, Chastang C, Boudiaf M, Le Corre A, Raskine L, Caulin C. Relevance in the emergency department of a decisional algorithm for outpatient care of women with acute pyelonephritis. Eur J Emerg Med 1999;6:15-20.

37) Tenner SM, Yadven MW, Kimmel PL. Acute pyelonephritis - preventing complications through prompt diagnosis and proper therapy. Postgrad Med 1992;91:261-8 